\title{
Neurophysiological correlates of visuospatial attention and the social dynamics of gaze processing
}

\author{
Grace Wei $^{1,2}$. Jacqueline A. Rushby ${ }^{1}$. Frances M. De Blasio ${ }^{1}$
}

Published online: 11 June 2019

(C) The Psychonomic Society, Inc. 2019

\begin{abstract}
The reflexive orienting response triggered by nonpredictive gaze cues is thought to be driven by a dedicated social neural network responsible for directing attention toward socially salient information. However, atypical processing of eye gaze using concomitant perceptual features has been proposed to underlie attentional orienting in groups with impairments in social cognition. Here, we examined the neurophysiological indices of visuospatial attention during a spatial cueing task, considering individual variability in social cognition in typically developing individuals, and the relative salience of social gaze and perceptual motion cues. We found enhanced neural activation to incongruent cues, wherein modulation of the N2b serves as a marker of the allocation of attention in the spatial domain. Our findings suggest the social gaze cue is less salient for those with greater autistic traits. An attentional bias toward perceptual motion cues correlated with greater social anxiety and alexithymia, and thus may reflect reduced sensitivity to social stimuli. These results provide evidence for likely neurophysiological mechanisms underlying gaze cueing and offer insight into the use of qualitatively different cognitive mechanisms used to access social information. Such paradigms provide potential insight into normative orienting responses reported in atypical groups and would benefit investigations of gaze following abilities in clinical populations.
\end{abstract}

Keywords EEG $\cdot$ ERP $\cdot$ Gaze cueing $\cdot$ PCA $\cdot$ Social cognition $\cdot$ Spatial attention

Discerning the basic neural processes that underlie social cognition and guide functional behaviour is fundamental to understanding the way in which we perceive and interact with the world around us. In consideration of the fluent reciprocity of social processes, the human eye in particular constitutes a unique visual stimulus in facilitating nonverbal communication (Bayliss et al., 2013). Unlike our nonhuman primate counterparts, the highly salient contrast between the sclera and iris of the human eye allows for the computation of gaze direction (Kobayashi \& Kohshima, 1997). Our eyes exist not only to see, as windows to the outside world, but are also

Electronic supplementary material The online version of this article (https://doi.org/10.3758/s13415-019-00728-w) contains supplementary material, which is available to authorized users.

Grace Wei

grace.wei@sydney.edu.au

1 School of Psychology, The University of New South Wales, Sydney, Australia

2 Present address: Brain and Mind Centre, University of Sydney, Camperdown, NSW 2050, Australia meant to be seen, providing an insight into one's mental state (Jarick \& Kingstone, 2015).

\section{The gaze-cueing effect}

Detecting and interpreting gaze information provides an insight into the minds of others, wherein their focus of attention has important implications for their knowledge, thoughts and desires (Frith \& Frith, 2006). In the context of social communication, studies of eye gaze have largely focused on the phenomenon of gaze cueing, wherein covert shifts of attention are elicited by a corresponding direction of gaze. Findings in the literature offer robust behavioural evidence whereby an attentional orienting response (i.e., target detection; reaction time) has been reliably shown to be faster to congruent relative to incongruent gaze information, even when the gaze cue is entirely nonpredictive of the target location (Driver et al., 1999; Friesen \& Kingstone, 1998).

This spontaneous gaze following triggered by uninformative gaze cues (i.e., gaze-cueing effect) suggests the special status of eye gaze as a stimulus of social and biological significance. In this way, it has been suggested that there exists a unique module for processing social cues responsible for 
prioritising social information independent from perceptual input (Baron-Cohen, 1995; Emery, 2000; Ristic et al., 2005). This dedicated means of processing gaze cues is thought to reflect the existence of specialised neural systems for social cognition, designed to enable quick and direct access to social information (Friesen \& Kingstone, 1998).

A distributed network of functionally relevant brain regions has been implicated in the computation of social stimuli, often conceptualised as the 'social brain' (Brothers, 1990). Activation of the same brain regions involved in processing eye gaze have also been found to be involved in tasks requiring the attribution of others' intentions and beliefs, therefore supporting the proposed relationship between gaze processing and theory of mind capacities (Calder et al., 2002; Castelli, Frith, Happe, \& Frith, 2002). As such, this notion of the social salience of gaze information underpinning the gaze-cueing effect endorses the existence of a specialised neural network mediating social cognition.

\section{Insights from autism}

In exploring the proposed 'social brain' network, it is therefore of particular interest to consider populations with deficits in social cognition. Qualitative impairments in social interaction and communication are one of the core characteristics of Autism spectrum disorders (ASDs; American Psychiatric Association, 2013). Despite impairments in gaze following in naturalistic settings, the majority of prior research provides strong evidence for intact gaze-cueing effects in autism (Leekam, Baron-Cohen, Perrett, Milders, \& Brown, 1997; Okada, Sato, Murai, Kubota, \& Toichi, 2003; Swettenham, Condie, Campbell, Milne, \& Coleman, 2003). Thus, the seemingly normal patterns of attentional orienting to eye gaze suggest an irreproducibility of this attribute of autism in structured laboratory settings.

Given that nonbiological cues (e.g., arrows) elicit similar behavioural responses, are reflexive shifts in attention in gazecueing paradigms specifically driven by dedicated social mechanisms? (Friesen, \& Kingstone, 2002; Hommel, Pratt, Colzato, \& Godijn, 2001; Nation \& Penny, 2008; Tipples, 2002) In light of this, it is possible that qualitatively different cognitive mechanisms may subserve the processing of eyegaze stimuli in these tasks. It has been suggested that the use of low-level perceptual features, such as visual or motion transients, facilitate access to social information in autism (Hayward \& Ristic, 2017; Ristic et al., 2005). Hayward and Ristic (2017) demonstrated that individuals with low social competence appear to be preferentially biased toward feature-based access to social information. In this way, the employment of compensatory strategies is thought to reflect a learned process (i.e., conditioning) whereby attention toward normally salient social cues, such as gaze, is mediated by spatial mechanisms. The notion that Posner-style cueing tasks (Posner, 1980) may be mediated by alternative processing strategies therefore offers a potential explanation for normative performance within clinical populations. Thus, wherein rudimentary gaze-following abilities remain intact, this may not necessarily require mental attribution nor involve any understanding of social significance (Leekam et al., 1997).

\section{Neurophysiological correlates of gaze cueing}

Fundamentally, neurophysiological methods are able to provide a measure of processing of stimuli that may be masked at a behavioural level. Specific event-related potential (ERP) components have been associated with visual attention. Conty, N'Diaye, Tijus, and George (2007) found that perception of direct gaze evoked a greater N170 than averted gaze, suggesting that in the early stage of gaze-direction processing, direct eye contact may trigger distinct social processes (Conty et al., 2007). Notably, robust links have been established between the N2 component and spatial selection in visual search and attentional cueing paradigms (Buodo, Sarlo, \& Munafò, 2010; Dell'Acqua, Sessa, Jolicoeur, \& Robitaille, 2006; Holmes, Bradley, Kragh Nielsen, \& Mogg, 2009). In the context of eye gaze, an increased gaze-cueing effect and thus expectancy of target onset at the cued location may be reflected in a more pronounced N2 response when such expectations are violated (i.e., incongruent trials; Galfano et al., 2011).

\section{The present study}

The aims of this study were twofold. The first was to identify the neurophysiological indices of the attentional dynamics of gaze cueing, extending upon previous behavioural findings (Hayward \& Ristic, 2017). It was hypothesised that the N2 component amplitude would be larger in incongruent trials relative to congruent trials, reflecting the functional dynamics of the N2 as a marker of the allocation of attention in the spatial domain (Eimer, 1996; Luck \& Hillyard, 1994). This was expected to parallel behavioural orienting responses.

Secondly, we aimed to investigate individual variability in gaze cueing. Here, we assessed the manipulation of stimulus motion as an attentional cue (Hayward \& Ristic, 2017). This involved the examination of social gaze cues, from direct to averted, as in the traditional gaze-cueing paradigm, and spatial cues involving motion transients. Two types of spatial cues were presented: mouth motion, being the displacement of the mouth line, and eye motion. Critically, as the eye motion trials involved the reversed movement sequence, from an averted to a direct gaze, they were incompatible with the construct of joint attention, despite maintaining the same perceptual feature (i.e., degree of motion). The task stimuli and trial sequence are 
illustrated in Fig. 1, with the experimental manipulation emphasised in the right panel for the three cue conditions. In this way, cueing effects underpinning attentional orienting to both gaze and spatial cues could be differentiated.

It is apparent that alternative strategies may be adopted in traditional gaze cueing paradigms, potentially masking gaze processing abnormalities (Hayward \& Ristic, 2017; Ristic \& Kingstone, 2012; Ristic \& Landry, 2015; Senju, Tojo, Dairoku, $\&$ Hasegawa, 2004). Here, we considered the multifaceted nature of social functioning in an attempt to explore the underlying mechanisms of social dysfunction. Notably, behavioural phenotypes found in autism are mirrored in individuals with social anxiety - specifically, an aversion to the eye region in face processing (Horley, Williams, Gonsalvez, \& Gordon, 2004; Moukheiber et al., 2010). The regulation of excessive fear through active avoidance behaviour may contribute to failures in extracting socially meaningful information from eye gaze (American Psychiatric Association, 2013; Horley, Williams, Gonsalvez, \& Gordon, 2003, 2004). Further, the consideration of alexithymia, a construct characterised by marked dysfunction in emotional awareness and interpersonal relating, may highlight a separate unique subset of social cognitive impairments (Bird, Press \& Richardson, 2011). Of interest here, alexithymia is highly prevalent in ASD (Berthoz, Hill, \& Hill, 2005). In contrast to active avoidance, as in social anxiety, alexithymia can be conceptualised as an inability or deficiency in social processing, thus reflecting a limitation rather than defence mechanism.

We hypothesised that individuals with higher social functioning (i.e., those with low alexithymic, autistic-like traits, and social anxiety) would show an increased gaze-cueing effect in the accelerated processing of social gaze relative to spatial cues. This preferential orienting of attention supports an 'interactive specialisation' of the social brain network, wherein advantages in neural processing reflect reductions in cognitive load in response to socially relevant input relative to spatial stimuli (Farroni, Csibra, Simion, \& Johnson, 2002; Johnson et al., 2005). Conversely, it was expected that an attentional bias towards perceptual changes (i.e., eye and mouth motion) in individuals with lower social functioning would reflect the use of, and sensitivity towards, low-level visual transients.

\section{Method}

\section{Participants}

Twenty-six undergraduate students $\left(M_{\text {age }}=19.3, S D_{\text {age }}=1.0\right.$ years; 23 right-handed) enrolled at the University of New South Wales participated in this study in exchange for course credit. Accounting for ASD and autistic-like trait sex bias and prevalence (Baron-Cohen, 2003; Halladay et al., 2015; Werling \& Geschwind, 2013), 20 participants were male and six were female. All reported normal or corrected-to-normal vision and no current or previous history of neurological and/or developmental disorders, substance dependence, or sensory problems. Participants were required to refrain from caffeine intake for at least 2 hours prior to the testing session (Barry, Clarke, Johnstone, \& Rushby, 2008; Barry et al., 2005). The experimental procedure was explained and written informed consent was obtained from all participants in accordance with the University of New South Wales Human Research Ethics Committee.

\section{Electrophysiological recording}

Continuous EEG was recorded from 64 scalp sites using a Ag/ $\mathrm{AgCl}$ electrode cap, referenced to the nose, and grounded by the cap electrode situated in the midline between Fz and FPz. Electrooculogram (EOG) data were recorded via electrodes placed $2 \mathrm{~cm}$ above and below the left eye (vertical), and via electrodes placed on the outer canthus of each eye

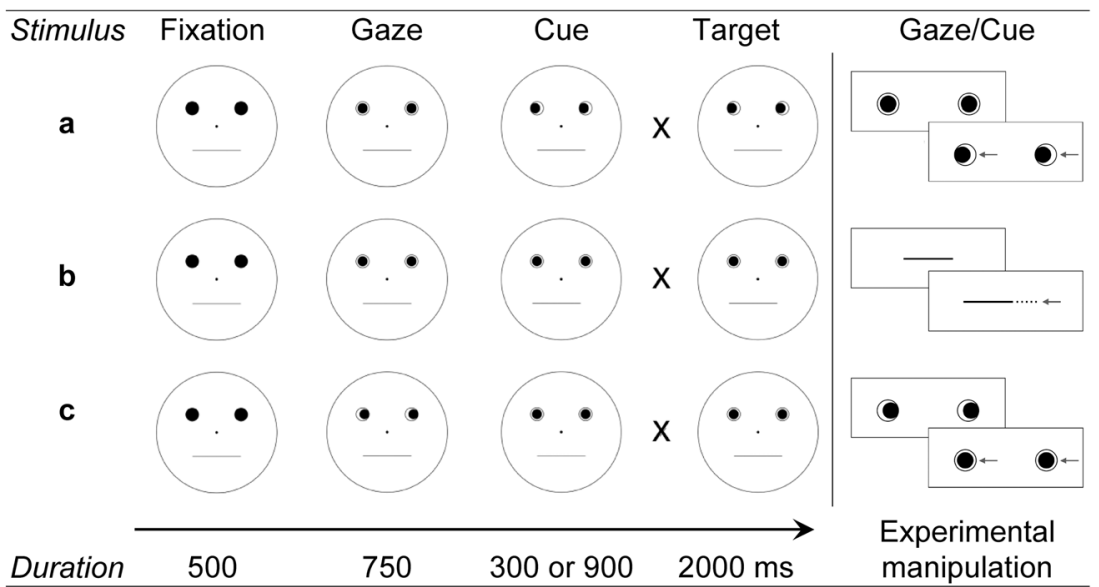

Fig. 1 Spatial cueing task. Trial sequence (left) and cue manipulation (right) are illustrated for the (a) social gaze, (b) mouth motion, and (c) eye motion cue conditions 
(horizontal). EEG data were sampled at $1000 \mathrm{~Hz}$ and recorded for offline analysis using Neuroscan Acquire software (Compumedics, Version 4.3). Care was taken to ensure impedances were below $5 \mathrm{k} \Omega$ for scalp, EOG, and reference electrodes.

\section{Procedure}

Participants were fitted with the EEG recording equipment and seated in an air-conditioned, dimmed, and soundattenuated recording room separate from the EEG amplifier.

They were then engaged in a computer task involving a Posner-style spatial cueing paradigm. Lastly, participants completed three questionnaires, in a counterbalanced order, to assess their social cognitive functioning.

\section{Spatial cueing task}

Analogous to prior studies, schematic faces were used to elicit gaze and spatial cueing (Friesen \& Kingstone, 1998; Hayward $\&$ Ristic, 2017); Figure 1 illustrates the trial sequence and example stimuli for each condition. Trials began with a fixation display with the onset of a face stimulus, presented centrally, composed of a circle outline (measuring $16.1^{\circ}$ of visual angle), filled eye outlines $\left(1.9^{\circ}\right)$, nose $\left(0.5^{\circ}\right)$ and mouth line $\left(6.7^{\circ}\right)$. This was followed by a straight (social gaze and mouth motion cue conditions) or averted (eye motion cue condition) gaze as denoted by the location of the pupil $\left(1.1^{\circ}\right)$ relative to the eye outline. The cue was then displayed for a randomly variable time interval (300 ms or $900 \mathrm{~ms}$ ), and its manipulation (relative to the preceding stimulus) is highlighted in the right panel for each condition; this involved the shift $\left(1.3^{\circ}\right)$ in gaze in the (Fig. 1a) social gaze and (Fig. 1c) eye motion conditions, or the displacement of the mouth line $\left(1.3^{\circ}\right)$ in the (Fig. 1b) mouth motion condition. The response target was a capital letter ' $X$ ' $\left(1.5^{\circ}\right)$ presented either to the left or right of the central fixation $\left(13^{\circ}\right.$ away), along the horizontal meridian, and remained on-screen for 2,000 ms irrespective of the participant's response. Stimuli were presented against a white background on a computer monitor (22-in. Alienware LCD Monitor) at an approximate distance of $60 \mathrm{~cm}$, using Presentation 18.3 software (Neurobehavioral Systems Inc.).

During the task, participants were instructed to minimise movement and to remain as relaxed as possible whilst completing the computer tasks. No fixation instructions were given. Participants were instructed to respond to the onset of the target stimuli ' $X$ ' as quickly and as accurately as possible by pressing the space bar. Trials were presented equally often for each condition (social gaze, mouth motion, eye motion), and within the conditions the combinations of target position (left, right) and trial type (congruent, incongruent) were balanced. Participants completed a total of 624 trials, and these were presented in a randomised order across four blocks with brief rest periods interspersed. Note that $\sim 8 \%$ of trials were presented without a target stimulus; participants were instructed to refrain from responding in these trials, and this served as a way to monitor and maintain the participant's attention.

\section{Psychometric assessment}

\section{Autism Spectrum Quotient}

The Autism Spectrum Quotient (AQ) is a 50-item self-report measure of autistic traits, wherein responses are made on a 4point Likert scale: 'strongly agree', 'slightly agree', 'slightly disagree' and 'strongly disagree'. Higher scores denote the greater presence of autistic traits. This instrument has been used in numerous studies of visual processing, validating the assumption that autistic-like traits exist along a continuum even within the typically developing (nonclinical) population (Nixima, Fujimori, \& Okanoya, 2013).

\section{Liebowitz Social Anxiety Scale}

The Liebowitz Social Anxiety Scale (LSAS-SR) is a 24-item self-report measure for the evaluation of social anxiety disorder, assessing fear and avoidance in 24 performance or interaction-based situations likely to elicit social anxiety (Liebowitz, 1987). This instrument demonstrates high internal consistency, strong convergent and discriminant validity, and is highly correlated with other measures of social anxiety disorder, including the clinician administered LSAS (Fresco et al., 2001). As such, this scale is considered to act as a reliable state measure of social anxiety.

\section{Toronto Alexithymia Scale}

The Toronto Alexithymia Scale (TAS-20) is a 20-item selfreport measure theoretically congruent with the alexithymia construct, composed of three subscales; Difficulty Describing Feelings, Difficulty Identifying Feelings, and ExternallyOriented Thinking (Bagby, Taylor, \& Parker, 1994). Responses are made on a 5-point Likert scale where $1=$ 'strongly disagree' and 5 = 'strongly agree'. Higher scores are indicative of greater difficulties in the corresponding alexithymia dimension/s. This measure is widely used and has been validated cross-culturally in both clinical and nonclinical samples (Timoney \& Holder, 2013).

\section{ERP quantification and analysis}

Continuous EEG data were band-pass filtered $(0.1-30 \mathrm{~Hz}$, zero-phase shift) and EOG corrected (Semlitsch, Anderer, Schuster, \& Presslich, 1986) in the Curry Neuroimaging Suite (Compumedics, Version 7.0.9 SBA). Waveforms for each of the trial types (congruent, incongruent) in each 
condition (social gaze, mouth motion, eye motion) were then epoched ( $-200 \mathrm{~ms}$ to $+1,000 \mathrm{~ms}$ relative to target onset), and baselined ( $-200 \mathrm{~ms}$ to $0 \mathrm{~ms})$. Trials with incorrect responses and/or extreme RT (RT beyond $M_{\mathrm{RT}} \pm 2 S D_{\mathrm{RT}}$ within-subjects) were identified and excluded from further analysis. An automatic artefact rejection procedure excluded additional trials if at any time during the epoch scalp activity exceeded $\pm 100 \mu \mathrm{V}$, or if the difference between successive data points exceeded $50 \mu \mathrm{V}$. The remaining trials were then averaged (within subjects) to form average ERPs for each trial type and condition. After correction, the following numbers were included per condition: gaze trials; congruent: $72.62 \pm 14.81$, incongruent: $71.81 \pm 14.46$; mouth motion trials; congruent: $74 \pm 13.35$, incongruent: $72.81 \pm 14.22$; eye motion trials; congruent: $72.96 \pm 14.93$, incongruent: $72.38 \pm 14.11$.

\section{Principal components analysis}

Separate (Barry, De Blasio, Fogarty, \& Karamacoska, 2016) temporal principal components analyses (PCAs) were conducted using the ERP PCA toolkit (Version 2.53; Dien, 2010) in MATLAB (The MathWorks, R2016a), one for each trial type (congruent, incongruent), for each condition (social gaze, mouth motion, eye motion). The ERP data were first down-sampled to $500 \mathrm{~Hz}$, and cut to $100-300 \mathrm{~ms}$; this period was chosen as it separated the components of interest (i.e., the N2) most effectively. Data for each PCA included 100 time points (variables) and 3,120 cases ( 26 participants $\times 2$ conditions $\times 60$ channels), used the covariance matrix with Kaiser normalisation, and all 100 factors underwent unrestricted Varimax rotation (Kayser \& Tenke, 2003).

PCA factors were identified as ERP components and retained for analysis based on their latency, polarity, topographic distribution, and variance accounted for. Within each condition (social gaze, mouth motion, eye motion), the similarity between corresponding components identified in each trial type (congruent, incongruent) were assessed using the congruence coefficient (Tucker, 1951) $\left(r_{c}\right)$, and associated interpretation guidelines (fair similarity: $.85 \leq r_{c} \leq .94$; equality: $r_{c}>.95$; Lorenzo-Seva \& ten Berge, 2006); only those components showing fair or greater similarity were analysed (i.e., $r_{c} \geq .85$ ).

\section{Statistical analyses}

All analyses were conducted in SPSS (IBM, Version 23), and $\alpha=.05$ was used for statistical significance. RT data were separately assessed for each condition (social gaze, mouth motion, eye motion) via repeated-measures MANOVAs with the within-subjects factor of trial type (congruent, incongruent).

The PCA-derived ERP component amplitudes were also assessed using separate repeated-measures MANOVAs.
The within-subjects factors were trial type (congruent, incongruent), and the sagittal (frontal, central, parietal) and lateral (left, midline, right) topographic dimensions; note that the positive and negative aspects of complex components were separately assessed within their maximal sagittal region, with trial type and laterality as the withinsubjects factors. Across these topographic dimensions, the contributing sites for each region were: frontal left (FL: FP1, AF3, F3, F5, F7), frontal midline (FM: FPz, $\mathrm{F} 1, \mathrm{Fz}, \mathrm{F} 2$ ), frontal right (FR: FP2, AF4, F4, F6,F8), central left (CL: FT7, FC5, FC3, T7, C5, C3, TP7, CP5, $\mathrm{CP} 3)$, central midline (CM: $\mathrm{FC} 1, \mathrm{FC}, \mathrm{FC} 2, \mathrm{C} 1, \mathrm{Cz}, \mathrm{C} 2$, $\mathrm{CP} 1, \mathrm{CPz}, \mathrm{CP} 2)$, central right (CR: $\mathrm{FC} 4, \mathrm{FC} 6, \mathrm{FT} 8, \mathrm{C} 4$, C6, T8, CP4, CP6, TP8), posterior left (PL: P7, P5, P3, $\mathrm{PO} 7, \mathrm{PO} 5, \mathrm{PO} 3, \mathrm{O} 1)$, posterior midline (PM: P1, Pz, P2, $\mathrm{POz}, \mathrm{Oz}$ ), and posterior right (PR: P4, P6, P8, PO4, PO6, $\mathrm{PO} 8, \mathrm{O} 2)$. Planned polynomial contrasts for the sagittal plane compared the frontal $(\mathrm{F})$ versus posterior $(\mathrm{P})$ regions, and the central region $(\mathrm{C})$ versus the frontalposterior mean $(\mathrm{F} / \mathrm{P})$. In the lateral plane, these compared the left $(\mathrm{L})$ versus right $(\mathrm{R})$ hemisphere, and the midline (M) versus hemispheric mean (L/R). As each contrast was planned a priori, and there were fewer of them than the degrees of freedom for effect, no Bonferroni-type adjustment to $\alpha$ was required (Tabachnik \& Fidell, 2013).

The intercorrelations between scores on each psychometric measure of social cognitive functioning (AQ, LSAS-SR, and TAS-20), and the associations between each of these measures and the magnitudes of the ERP and RT trial congruency processing costs were assessed using bivariate correlations. The trial congruency processing costs were quantified (within subjects) as the difference between the incongruent and congruent trial data (i.e., incongruent-congruent) in each outcome measure showing a significant effect involving trial type (congruent vs. incongruent) in the aforementioned RT and ERP analyses (see Table 1). Note that the trial congruency processing costs in the ERP components were assessed for the global (main effect) or relevant regional (interaction effect) acrosssite mean/s. No corrections for multiple testing were applied (Bender \& Lange, 2001). Although exploratory in nature, these analyses were guided by the statistical outcomes in Table 1. Here, the relationships between the psychometric measures and the incongruence processing cost indices were quite specific in their patterning, despite the intercorrelations between the psychometric measures.

\section{Results}

\section{Congruency effects in behavioural responding}

Mean reaction time (RT) data are displayed in Fig. 2, and the associated statistics reported in Table 1. Target RT was 


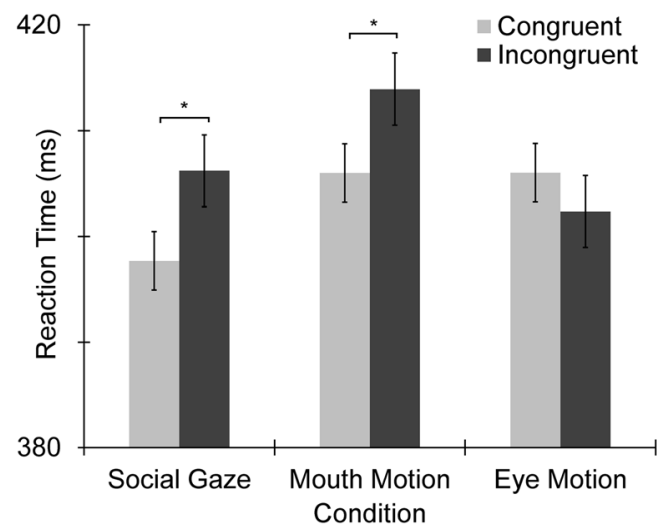

Fig. 2 Behavioural outcomes. Mean RT is plotted as a function of cue/ target congruency (trial type) in each cue condition. Error bars indicate standard error, and significant effects $(p<.05)$ are indicated with an asterisk and bar above the relevant cue condition

significantly delayed for incongruent $(406.20 \mathrm{~ms} \pm 97.71 \mathrm{~ms})$ relative to congruent $(397.68 \mathrm{~ms} \pm 96.13 \mathrm{~ms})$ trials in the social gaze condition $(p=.021)$. Target RT was also significantly delayed for incongruent $(413.93 \mathrm{~ms} \pm 99.23 \mathrm{~ms})$ relative to congruent $(405.99 \mathrm{~ms} \pm 99.59 \mathrm{~ms})$ trials in the mouth motion condition ( $p=.049)$. RT did not differ significantly between the incongruent (402.36 ms $\pm 94.14 \mathrm{~ms})$ and congruent (406.01 ms $\pm 100.27 \mathrm{~ms})$ trials in the eye motion condition $(p=.195)$.

\section{ERP component quantification}

Figure 3 illustrates the grand mean ERPs (across participants and sites) time-locked to target onset for the congruent and incongruent trial types in the social gaze (Fig. 3a), mouth motion (Fig. 3b), and eye motion (Fig. 3c) cue conditions. The latency range entered into each PCA (i.e., 100-300 ms) are also indicated, and within this range $\mathrm{P} 2$ and $\mathrm{N} 2$ components are prominent at approximately $150-160 \mathrm{~ms}$ and 200 $210 \mathrm{~ms}$, respectively. The corresponding PCA outcomes are presented in Fig. 4. Two components were identified in each of the trial types (congruent, incongruent) for the social gaze (Fig. 4a) and mouth motion (Fig. 4b) cue conditions, while three were similarly identified in the eye motion (Fig. 4c) condition; refer to Fig. 4 for the variance and latency information for each identified component. Temporally, the first component in each data set was identified as a complex consisting of a frontal processing negativity (PN; Näätänen, Gaillard, \& Mantysalo, 1978) and parietal P2, here labelled the PN/P2 complex, and this was followed by an $\mathrm{N} 2 \mathrm{~b}$. The additional component in the eye motion cue condition followed the $\mathrm{N} 2 \mathrm{~b}$ and was identified as the N2c. Congruence coefficients indicated high similarity or component equality between the corresponding components in the congruent and incongruent trial types for each cue

Table 1 Behavioural and ERP outcomes in each cue condition

\begin{tabular}{|c|c|c|c|c|c|c|}
\hline Condition & & Measure & Effect & $F$ & $p$ & $\eta_{\mathrm{p}}^{2}$ \\
\hline \multirow[t]{8}{*}{ a } & \multirow[t]{8}{*}{ Social gaze } & RT & Cong $<$ Incong & 6.04 & .021 & .19 \\
\hline & & Frontal PN & $\mathrm{M}>\mathrm{L} / \mathrm{R}$ & 5.90 & .023 & .19 \\
\hline & & Posterior P2 & $\mathrm{M}<\mathrm{L} / \mathrm{R}$ & 4.54 & .043 & .15 \\
\hline & & & Cong $<$ Incong $\times \mathrm{M}>\mathrm{L} / \mathrm{R}$ & 6.60 & .017 & .21 \\
\hline & & $\mathrm{N} 2 \mathrm{~b}$ & $\mathrm{C}>\mathrm{F} / \mathrm{P}$ & 5.46 & .028 & .18 \\
\hline & & & $\mathrm{L}<\mathrm{R}$ & 8.02 & .009 & .24 \\
\hline & & & $\mathrm{M}>\mathrm{L} / \mathrm{R}$ & 11.97 & .002 & .32 \\
\hline & & & Cong $<$ Incong $\times \mathrm{F}>\mathrm{P}$ & 15.37 & .001 & .38 \\
\hline \multirow[t]{5}{*}{$\mathrm{b}$} & \multirow[t]{5}{*}{ Mouth motion } & RT & Cong $<$ Incong & 4.28 & .049 & .15 \\
\hline & & Posterior P2 & $M>L / R$ & 4.66 & .041 & .16 \\
\hline & & $\mathrm{N} 2 \mathrm{~b}$ & $\mathrm{M}>\mathrm{L} / \mathrm{R}$ & 5.21 & .031 & .17 \\
\hline & & & Cong $<$ Incong $\times \mathrm{M}>\mathrm{L} / \mathrm{R}$ & 4.41 & .046 & .15 \\
\hline & & & Cong $<$ Incong $\times \mathrm{F}<\mathrm{P} \times \mathrm{M}>\mathrm{L} / \mathrm{R}$ & 4.29 & .049 & .15 \\
\hline \multirow[t]{13}{*}{$\mathrm{c}$} & \multirow[t]{13}{*}{ Eye motion } & $\mathrm{N} 2 \mathrm{~b}$ & $\mathrm{~F}>\mathrm{P}$ & 10.11 & .004 & .29 \\
\hline & & & $\mathrm{C}>\mathrm{F} / \mathrm{P}$ & 7.95 & .009 & .24 \\
\hline & & & $M>L / R$ & 16.59 & $<.001$ & .40 \\
\hline & & & $\mathrm{F}>\mathrm{P} \times \mathrm{M}<\mathrm{L} / \mathrm{R}$ & 6.82 & .015 & .21 \\
\hline & & & Cong $<$ Incong & 8.25 & .008 & .25 \\
\hline & & & Cong $<$ Incong $\times \mathrm{F}<\mathrm{P}$ & 4.31 & .048 & .15 \\
\hline & & & Cong $<$ Incong $\times \mathrm{M}>\mathrm{L} / \mathrm{R}$ & 9.85 & .004 & .28 \\
\hline & & & Cong $<$ Incong $\times \mathrm{F}<\mathrm{P} \times \mathrm{M}>\mathrm{L} / \mathrm{R}$ & 4.71 & .040 & .16 \\
\hline & & $\mathrm{N} 2 \mathrm{c}$ & $\mathrm{F}<\mathrm{P}$ & 5.69 & .025 & .19 \\
\hline & & & $\mathrm{C}<\mathrm{F} / \mathrm{P} \times \mathrm{L}>\mathrm{R}$ & 4.88 & .037 & .16 \\
\hline & & & Cong $<$ Incong $\times \mathrm{C}<\mathrm{F} / \mathrm{P}$ & 5.87 & .023 & .19 \\
\hline & & & Cong $<$ Incong $\times \mathrm{M}<\mathrm{L} / \mathrm{R}$ & 13.32 & .001 & .35 \\
\hline & & & Cong $<$ Incong $\times \mathrm{F}<\mathrm{P} \times \mathrm{M}<\mathrm{L} / \mathrm{R}$ & 11.20 & .003 & .31 \\
\hline
\end{tabular}

Note. Each $F$ test had $(1,25)$ degrees of freedom.

$\mathrm{F}=$ frontal $\mathrm{C}=$ central $; \mathrm{P}=$ posterior $; \mathrm{F} / \mathrm{P}=$ frontoparietal mean $\mathrm{L}=$ left hemisphere; $\mathrm{M}=$ midline; $\mathrm{R}=$ right hemisphere; $\mathrm{L} / \mathrm{R}=$ hemispheric mean; Cong = congruent cue/target trial type; Incong = incongruent cue/target trial type 


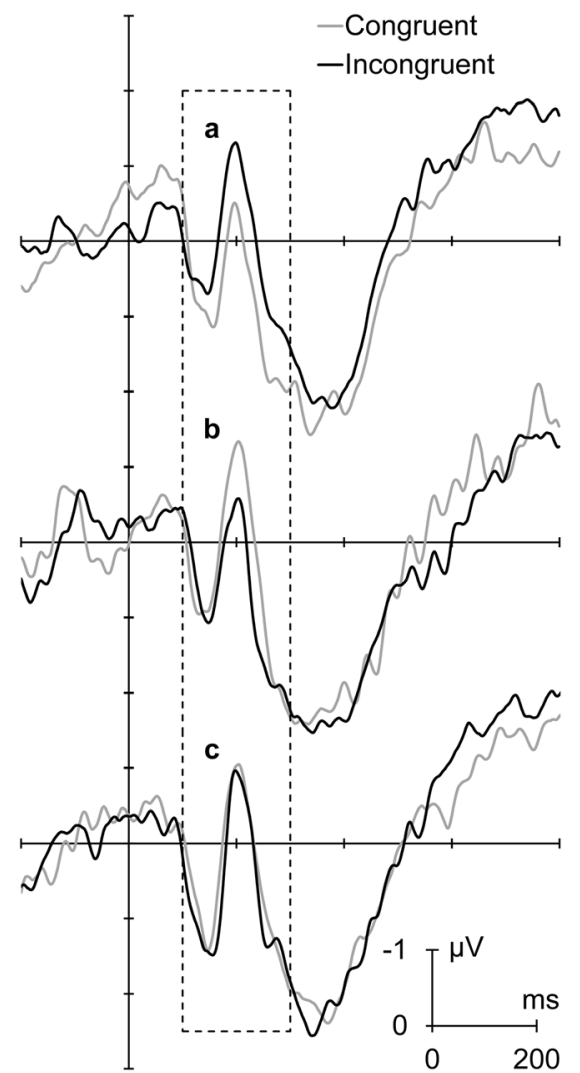

Fig. 3 Grand mean ERPs time-locked to target onset, across participants and sites. Data are shown for the congruent and incongruent cue/target trial types in each of the (a) social gaze, (b) mouth motion, and (c) eye motion cue conditions. The dashed boxes identify the period entered into each PCA condition (all $r_{c} \geq .92$ ), and thus each were further assessed (Lorenzo-Seva \& ten Berge, 2006).

\section{Component topographies and congruency effects}

Table 1 displays statistics for the grand mean component topographies and significant effects and interactions involving trial type for each cue condition. These effects are illustrated in the Fig. 4 grand mean (GM) and difference (incongruent-congruent) head maps, respectively.

\section{Social gaze}

The grand mean frontal PN was maximal in the midline, and did not show an effect of trial type. The posterior P2 was reduced in the midline than in hemispheres across trial types, although it was larger in the midline for incongruent relative to congruent trials. $\mathrm{N} 2 \mathrm{~b}$ in the social gaze condition was relatively larger in the central and midline regions, and was reduced in the left relative to right hemisphere across trial types. N2b was enhanced frontally for incongruent relative to congruent trials.

\section{Mouth motion}

The frontal PN did not show any significant topographic or trial congruency effects, while the posterior P2 was greater in the midline than hemispheres and did not differ with trial type. $\mathrm{N} 2 \mathrm{~b}$ was midline dominant across the trial types, and this

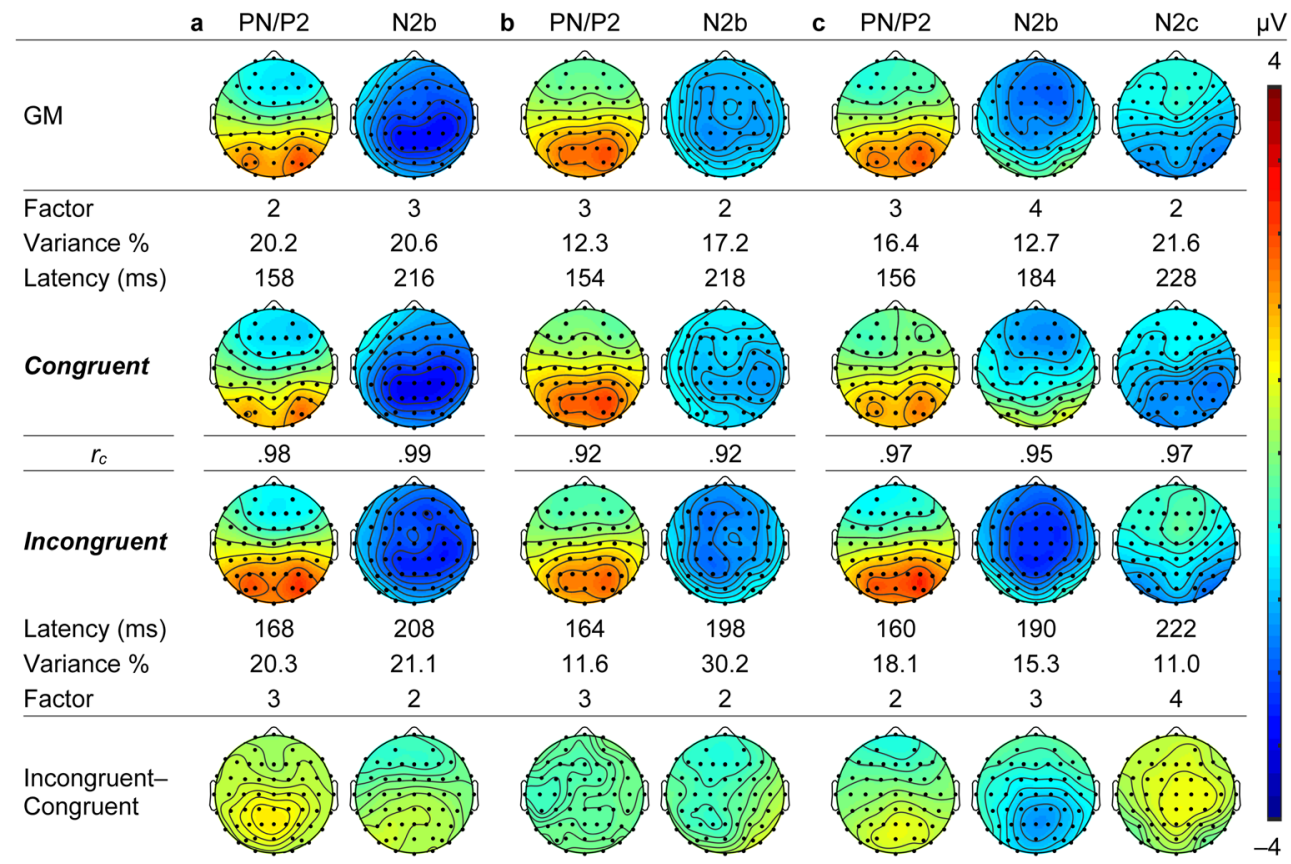

Fig. 4 PCA-derived ERP component outcomes. Topographic plots and factor information for the identified ERP components in the congruent and incongruent cue/target trial types, their grand mean (GM), and difference (incongruent relative to congruent), for each of the (a) social gaze, (b) mouth motion, and (c) eye motion cue conditions. (Colour figure online) 
topography was enhanced for incongruent relative to congruent trials, particularly in the posterior region.

\section{Eye motion}

Neither the frontal PN nor posterior P2 showed significant effects of topographic laterality or trial congruency. Across trial types, $\mathrm{N} 2 \mathrm{~b}$ was dominant in the frontocentral, midline, and frontalhemispheric regions. In the incongruent (cf. congruent) trials, $\mathrm{N} 2 \mathrm{~b}$ was enhanced globally across the assessed regions, posteriorly, in the midline, and in the posterior midline. N2c was greater in the posterior than frontal region, and reduced in the central left across trial types. N2c was enhanced in the posterior hemispheres for incongruent than for congruent trials, but showed relative reductions in the central and midline regions in this trial type.

\section{Social cognition measures and their association with congruency processing}

Significant bivariate correlations between the psychometric measures (AQ, LSAS-SR, TAS-20) are displayed at the top of Table 2; significant and direct (cf. inverse) intercorrelations were found between each measure (see Supplementary Fig. $\mathrm{S} 1$ ). Correlations between the magnitude of the incongruence processing cost in each condition (social gaze, mouth motion, eye motion) for each measure (RT and ERP) showing significant congruency effect in Table 1 , and the social cognition measures are presented in the bottom of Table 2. The social cognition measures each did not correlate with incongruence RT processing costs in the social gaze and mouth motion conditions, nor with the incongruence ERP processing costs indexed via regional $\mathrm{N} 2 \mathrm{~b}$ amplitudes in the social gaze condition, and via the global N2b amplitude and regional N2b and $\mathrm{N} 2 \mathrm{c}$ amplitudes in the eye motion condition.

Table 2 Psychometric score intercorrelations and association with ERP indices of incongruence processing costs

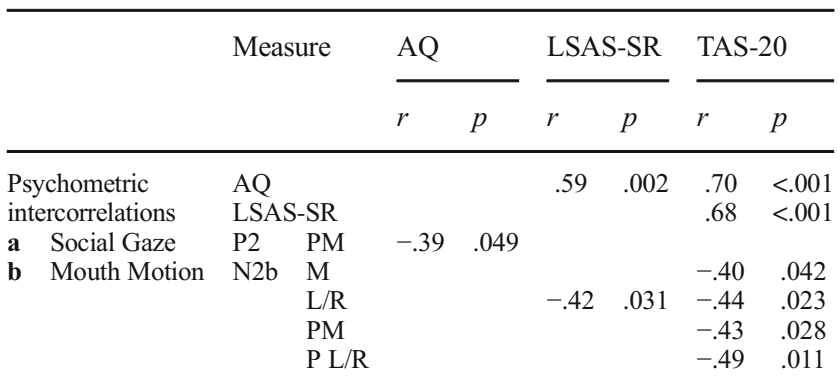

Note. ERP data are difference scores reflecting regional incongruence processing costs (i.e., incongruent-congruent peak component amplitudes). Only significant correlations are shown; all $r$ had 24 degrees of freedom and are reported with two-tailed significance.

\section{AQ}

Higher scores on the AQ were associated with smaller incongruence processing costs as indexed by the posterior midline P2 in the social gaze condition, an inverse linear trend. That is, posterior midline P2 amplitudes were greater in congruent than incongruent trials in those who scored higher on the AQ. This was the only marker of incongruence processing costs to show an association with the AQ.

\section{LSAS-SR}

LSAS-SR scores were associated with incongruence processing costs as indexed by the hemispheric N2b in the mouth motion condition. As this is a negative component, the inverse correlation indicates that higher LSAS-SR scores were associated with increased (i.e., more negative) hemispheric N2b incongruence processing costs. This was the only marker of incongruence processing costs to show an association with the LSAS-SR.

\section{TAS-20}

Scores on the TAS-20 correlated with several regional N2b markers of incongruence processing costs in the mouth motion condition. Each of these associations were inverse, and thus more negative N2b (i.e., increased) markers of incongruence processing costs in the midline, hemispheres, posterior midline, and posterior hemispheres were each associated with higher TAS-20 scores.

\section{Discussion}

This study aimed to identify the neurophysiological indices of the visuospatial attentional dynamics of gaze cueing, and to explore the relative salience of social and perceptual cues considering individual variability in social cognitive functioning. Using principal components analysis (PCA) to clarify the ERP components elicited by a gaze-cueing task, this study revealed electrophysiological differences in the processing of congruent relative to incongruent gaze cues. Specifically, in line with behavioural cueing effects, modulation of the $\mathrm{N} 2 \mathrm{~b}$ appeared to index the allocation of attention in the spatial domain. Results from this study further illustrated differences in the processing of social and spatial cues. Importantly, divergent profiles of social cognitive functioning emerge when considering the relative sensitivity to these cues. An attentional bias towards motion transients was found in those with greater social anxiety and alexithymic traits. How these findings inform the understanding of the mechanisms of gaze processing and shed light on the links between perceptual processes and social cognition are discussed below. 


\section{Neurophysiological correlates of gaze cueing}

As a time-locked measure of electrical activity in the brain, ERPs are particularly useful in understanding the nature and sequence of cognitive processes. With respect to quantifying the magnitude of amplitude modulations, traditional approaches considering voltage peaks (e.g., peak picking methods) are potentially misleading and uninformative. Measuring the voltage of the ERP waveform at its peak or trough imbues value in arbitrary local maxima wherein amplitude modulations between two conditions may be the result of contributions from the earlier offset of a previous component or the earlier onset of a later component (Lorenzo-Seva \& ten Berge, 2006; Näätänen et al., 1978; Näätänen \& Michie, 1979; Woodman, 2010). Here, the data-driven decomposition of the ERP waveform into its underlying components using PCA helped to clarify cognitive processing.

Two ERP components were elicited in the social gazecueing task within the specified latency range (100-300 ms). The topographic distribution of the first component extracted likely points to overlap of a frontal processing negativity (PN) defined by Näätänen et al. (1978) and posterior P2. The PN component has been posited to reflect the beginning of identification of the eliciting stimulus (Barry \& De Blasio, 2015). In this paradigm, PN component amplitudes did not differ for cue congruency. The functional dynamics of the P2 component shares in the representation of early sensory processing, believed to be sensitive to the physical characteristics of the stimulus. Consistent with this notion, the posterior P2 elicited here points to activity originating near primary cortical sensory areas in the visual modality (Goff, Allison, \& Vaughan, 1978; Vaughan \& Arezzo, 1998). Findings from tasks involving selective attention suggest that modulation of the P2 reflects attentional enhancement, observed in larger P2 amplitudes to attended stimuli (Hillyard \& Kutas, 1983). In line with the selective attention hypothesis, here, we found enhanced hemispheric dominance of the $\mathrm{P} 2$ in social gaze trials to the presentation of congruently cued targets in attended locations.

With regards to the $\mathrm{P} 2 / \mathrm{PN}$ component, a lack of stable cuebased interaction effects across different conditions mean that findings need to be interpreted with relative caution, wherein limited conclusions may be drawn regarding its functional significance in attentional cueing. Further, it has been previously suggested that the first extracted component, temporally defined here as the P2/PN, likely consists of 2 components; a frontal negativity and posterior positivity, overlapping in latency. As such, contributions specific to the P2 component may not be reliably separated from the PN.

The focus of the present investigation on the N2 component thus reflects research traditions highlighting facets of cognitive control as determinants of the N2 amplitude (Folstein \& Van Petten, 2008). The central cortical distribution of the latter component extracted appears to correspond to the anterior N2, labelled N2b, occurring during conscious stimulus attention (Pritchard, Shappell, \& Brandt, 1991). Findings from go/no-go studies support the notion that the $\mathrm{N} 2 \mathrm{~b}$ is driven by the immediate control of action, encompassing response inhibition, such that a larger N2b to the 'no-go' stimulus is posited to reflect the suppression of an incorrect response (Bruin \& Wijers, 2002; Pfefferbaum, Ford, Weller, \& Kopell, 1985). As hypothesised, in the gaze-cueing task, the N2b amplitude was greater in incongruent trials relative to congruent trials in the frontal regions. Here, modulation of the N2b amplitude may relate to increases in cognitive processing requirements associated with greater target stimulus deviance, reflecting the suppression of attentional shifts to incongruently cued locations. In line with behavioural gazecueing effects - that is, slower RTs to incongruent trials compared with congruent trials - enhanced frontal activation to incongruent gaze cues thus provides evidence for an inhibitory mechanism in response to expectancy violations, wherein the N2b may serve as a marker of the visuospatial attentional dynamics of gaze cueing, and, as such, providing a measure of top-down modulation of visual processing underlying the critical ability to guide attentional resources toward relevant stimuli. Notably, in the mouth motion condition, while a significant behavioural cueing effect was also observed, the $\mathrm{N} 2 \mathrm{~b}$ was enhanced posteriorly to incongruent trials. While the motion here exists within the context of a face (i.e., mouth), the cueing observed here may be more akin to that elicited by nonbiological arrow cues (Hietanen et al., 2006). These results support the notion of dissociable neural mechanisms underpinning attentional orienting to gaze and spatial cues.

The present study serves as a methodological bridge between traditional behavioural approaches and neurophysiological investigations (Hayward \& Ristic, 2017). Here, we used schematic faces to elicit gaze-cueing effects. In probing the neural mechanisms of gaze cueing, it is important that such initial investigations are founded on established, simple, and robust behavioural effects. That said, however, future investigations should work towards incorporating more contextually situated and ecologically valid procedures, considering dynamic paradigms and real faces, to improve the understanding of gaze processing and social attention dynamics in real world interactions. While the target stimulus was examined in the current study, other ERP components including the early attention directing negativity (EDAN), anterior attention directing negativity (ADAN), and late directing attention positivity (LDAP) elicited to the cue stimulus may further delineate the extent to which preparatory orienting and short-term memory processes are related to social cognitive activity (Lasaponara et al., 2018; Murray, Nobre, \& Stokes, 2011; Seiss, Gherri, Eardley, \& Eimer, 2007). 


\section{Visuospatial attention: Social and spatial cues}

The manipulation of stimulus motion in this study allowed for the examination of gaze and spatial attentional cueing (Hayward \& Ristic, 2017). As expected, a behavioural gazecueing effect was elicited in the social gaze condition, in line with the robust nature of attentional orienting to gaze cues (Friesen \& Kingstone, 1998). Importantly, despite maintaining the same perceptual feature of motion, spatial eye motion cues had no significant effect on attentional orienting (i.e., no reaction time differences between responses to congruent and incongruent cues). The disparity between these two conditions thus provides support for the differentiation of gaze and spatial cueing effects. In their relative compatibility with the construct of joint attention, the relevance of the social gaze cue lies in its sequencing from a direct to an averted gaze. These findings highlight the capacity of direct gaze to capture visual attention, reflecting its social significance in nonverbal communication and the establishment of communicative intent. While not significant, there is a trend to suggest that the inhibition of return (IOR) effect could account for the opposite direction of behavioural effects for the eye motion condition (Klein, 2000).

Neurophysiologically, although the lack of behavioural differences did not preclude an incongruency effect in the $\mathrm{N} 2 \mathrm{~b}$ component, this disparity was reflected in the presence of a third ERP component. The topography of the third extracted component, specific to the eye motion condition, appears to correspond to the posterior N2, labelled N2c (Pritchard et al., 1991). Modulations of this subcomponent of the N2 are thought to be related to the degree of response conflict, representing the amount of attention needed for classification of stimulus features within the visual cortex (Suwazono, Machado, \& Knight, 2000). As such, the enhancement of the N2c amplitude in the posterior hemispheres on incongruent trials may be indicative of increased processing demands and response conflict, whereby attention is divided between the salient direct gaze and the target.

Whereas the high contrast boundaries of eye gaze appear to dominate the attention system as a prioritised stimulus, this is perhaps limited to the ability of direct gaze to rapidly arouse and modulate the attention of the observer (Birmingham \& Kingstone, 2009; Strom \& Buck, 1979). The present findings thus extend upon the understanding of attentional orienting triggered by gaze cues by highlighting the specificity of the social significance of direct gaze.

\section{Patterns of visuospatial attention across profiles of social cognitive functioning}

By considering social anxiety and alexithymia, reflecting hypersensitivity and hyposensitivity to social stimuli respectively (Bird, Press, \& Richardson, 2011; Horley et al., 2003, 2004), the present study attempted to examine the underlying nature of deficits in social cognition alongside autistic-like traits more broadly. Thus, providing a more refined approach and clearer picture of the influence of related individual variables on patterns of human social attention.

It was hypothesised that patterns of visuospatial attention would differ as a function of individual variability in social cognition, reflecting differences in the processing of social information. Specifically, individuals with impairments in social cognitive functioning may access social information through spatial channels, reflected in a bias of attention towards low-level perceptual changes. In this study, patterns of visuospatial attention and susceptibility to cueing effects were examined by considering motion transients.

Differences in gaze and spatial (i.e., motion based) attentional orienting were examined in relation to profiles of social cognitive functioning. As this study examined the modulation of the $\mathrm{N} 2 \mathrm{~b}$ in attentional cueing, the reliability of functional interpretations of the elicited ERP component is taken into account in the subsequent consideration of the influence of social cognition. Here, the enhancement of the $\mathrm{N} 2 \mathrm{~b}$ to incongruent cues across conditions provides converging lines of evidence supporting the functional dynamics of the $\mathrm{N} 2 \mathrm{~b}$ as a marker of the allocation of attention in the spatial domain.

In the social gaze condition, higher scores on the AQ were correlated with smaller incongruence processing costs. Here, the contrasting directionality (i.e., reduced hemispheric dominance of the P2 in congruent trials) in those with higher AQ scores, suggests that the social gaze cue may be less salient in directing attention in those with greater autistic traits.

In line with the notion of reduced salience, in the mouth motion condition, both higher LSAS-SR and TAS-20 scores correlated with larger incongruence processing costs in the $\mathrm{N} 2 \mathrm{~b}$ component. Here, higher scores on these measuresthat is, greater social anxiety and alexithymia - were associated with greater response inhibition reflected in the $\mathrm{N} 2 \mathrm{~b}$ (i.e., larger N2b amplitudes to incongruent trials). Taken together, these data support the notion that individuals with impairments in social cognitive functioning may utilise concomitant low-level perceptual changes in the environment to access the social content from gaze cues. Specifically, greater social anxiety and alexithymic traits could account, at least in part, for attentional bias towards motion transients, reflected neurophysiologically in greater inhibition of spatial incongruently cued locations. The differing patterns of visuospatial attention offer support for preferentially biased attention to perceptual features used to access social information.

Given the nature of the participant pool as a nonclinical population, findings with respect to autistic-like traits are inherently more nuanced relative to atypical groups. However, these findings suggest that considering social anxiety and alexithymic traits may provide a better conceptualisation of social cognitive impairment, reflecting specific deficits in the representation of social stimuli. Such lines of inquiry may 
ultimately have profound implications for the identification of subgroups within clinical populations with specific patterns of deficits and allow for subsequent opportunities for individually adjusted training and intervention. Whilst conclusions drawn from this data are limited due to the nature and size of the study sample, such findings show prospective relevance to atypical populations. Future investigations may consider clinical groups with social cognitive impairments such as autism spectrum disorders, traumatic brain injury (TBI), and schizophrenia, wherein a lack of preferential sensitivity to social stimuli may underlie difficulties in social interaction. Notably, electrophysiological methodologies do not necessarily require a verbal or motor response, making them appropriate for populations with limited cognitive or communicative abilities.

\section{Conclusions}

In summary, we have demonstrated links between attentional biases, perceptual processes, and social functioning. This study grants novel insight into the intricate interplay of processes involved in gaze following and visuospatial attention and has theoretical relevance for models of gaze processing. From a clinical perspective, the consideration of concomitant perceptual features offers insight into normative performances seen in atypical groups. Crucially, the proposed lack of preferential sensitivity to social stimuli contributes to the understanding of deficits in higher-order social cognition and the flexibility with which to use gaze cues in other contexts. This failure to prioritise and appropriately interpret social gaze in turn compromises opportunities for learning and communication development via the experience of social interaction (Mundy, 1995). The development of such compensatory strategies may allow individuals to achieve normative performance in structured laboratory situations, however, may manifest in reduced social competence in a dynamic real-life context, whereby unpredictability and other features in the environment may ultimately contribute to impairments in social interaction. Whilst unimpaired at a perceptual level, this prolonged reliance on mechanistic access to social information adversely affects behavioural and functional domains dependent on these early processes, and thus, may explain the developmental trajectory of autism. This schema and paradigm provides a valuable framework for future investigations to improve understanding of the mechanisms of visuospatial attention and nature of social cognitive functioning across simple experimental and dynamic real-world contexts, in both typical and atypical populations.

Acknowledgements We thank Christopher Sufani for his assistance with stimulus development and data processing and Rebecca El Helou for her assistance in data collection. J.R. was supported by a National Health and Medical Research Council of Australia Project Grant (1081923).
Data availability The data sets generated during and/or analysed during the current study are available from the corresponding author on reasonable request.

\section{References}

American Psychiatric Association. (2013). Diagnostic and statistical manual of mental disorders (5th ed.). Washington, DC: Author.

Bagby, R. M., Taylor, G. J. \& Parker, J. D. (1994). The Twenty-Item Toronto Alexithymia Scale-II. Convergent, discriminant, and concurrent validity. Journal of Psychosomatic of Research, 38(1), 3340.

Baron-Cohen, S. (1995). Mindblindness: An essay on autism and theory of mind. Learning, development, and conceptual change. Cambridge, MA: MIT Press.

Baron-Cohen, S. (2003). The essential difference: Men, women and the extreme male brain. London, UK: Penguin Books.

Barry, R. J., Clarke, A. R., Johnstone, S. J., \& Rushby, J. A. (2008). Timing of caffeine's impact on autonomic and central nervous system measures: Clarification of arousal effects. Biological Psychology, 77(3), 304-316.

Barry, R. J., \& De Blasio, F. M. (2015). Performance and ERP components in the equiprobable go/no-go task: Inhibition in children. Psychophysiology, 52(9), 1228-1237.

Barry, R. J., De Blasio, F. M., Fogarty, J. S., \& Karamacoska, D. (2016). ERP go/no-go condition effects are better detected with separate PCAs. International Journal of Psychophysiology, 106, 50-64.

Barry, R. J., Rushby, J. A., Wallace, M. J., Clarke, A. R., Johnstone, S. J., \& Zlojutro, I. (2005). Caffeine effects on resting-state arousal. Clinical Neurophysiology, 116(11), 2693-2700.

Bayliss, A. P., Murphy, E., Naughtin, C. K., Kritikos, A., Schilbach, L., \& Becker, S. I. (2013). "Gaze leading”: Initiating simulated joint attention influences eye movements and choice behavior. Journal of Experimental Psychology: General, 142(1), 76-92.

Bender, R., \& Lange, S. (2001). Adjusting for multiple testing-when and how? Journal of Clinical Epidemiology, 54(4), 343-349.

Berthoz, S., \& Hill, E. L. (2005). The validity of using self-reports to assess emotion regulation abilities in adults with autism spectrum disorder. European Psychiatry, 20(3), 291-298.

Bird, G., Press, C., \& Richardson, D. C. (2011). The role of alexithymia in reduced eye-fixation in autism spectrum conditions. Journal of Autism and Developmental Disorders, 41(11), 1156-1164.

Birmingham, E., \& Kingstone, A. (2009). Human social attention: A new look at past, present, and future investigations. Annals of the New York Academy of Sciences, 1156, 118-140.

Brothers, L. (1990). The social brain: A project for integrating primate behaviour and neurophysiology in a new domain. Concepts in Neuroscience, 1, 27-51.

Bruin, K. J., \& Wijers, A. A. (2002). Inhibition, response mode, and stimulus probability: A comparative event-related potential study. Clinical Neurophysiology, 113(7), 1172-1182.

Buodo, G., Sarlo, M., \& Munafò, M. (2010). The neural correlates of attentional bias in blood phobia as revealed by the N2pc. Social Cognitive and Affective Neuroscience, 5, 29-38.

Calder, A. J., Lawrence, A. D., Keane, J., Scott, S. K., Owen, A. M., Christoeffels, I., \& Young, A. W. (2002). Reading the mind from eye gaze. Neuropsychologia, 40(8), 1129-1138.

Castelli, F., Frith, C., Happe, F., \& Frith, U. (2002). Autism, Asperger syndrome and brain mechanisms for the attribution of mental states to animated shapes. Brain, 125, 1839-1849.

Conty, L., N'Diaye, K., Tijus, C., \& George, N. (2007). When eye creates the contact! ERP evidence for early dissociation between direct and averted gaze motion processing. Neuropsychologia, 45, 3024-3037. 
Dell'Acqua, R., Sessa, P., Jolicoeur, P., \& Robitaille, N. (2006). Spatial attention freezes during the attentional blink. Psychophysiology, 43, 394-400.

Dien, J. (2010). The ERP PCA Toolkit: An open source program for advanced statistical analysis of event-related potential data. Journal of Neuroscience Methods, 187(1), 138-145.

Driver, J., Davis, G., Ricciardelli, P., Kidd, P., Maxwell, E., \& BaronCohen, S. (1999). Gaze perception triggers reflexive visuospatial orienting. Visual Cognition, 6(5), 509-540.

Eimer, M. (1996). The N2pc component as an indicator of attentional selectivity. Electroencephalography \& Clinical Neurophysiology, 99(3), 225-234.

Emery, N. J. (2000). The eyes have it: The neuroethology, function and evolution of social gaze. Neuroscience and Biobehavioral Reviews, 24(6), 581-604.

Farroni, T., Csibra, G., Simion, F., \& Johnson, M. H. (2002). Eye contact detection in humans from birth. Proceedings of the National Academy of Sciences of the United States of America, 99(14), 9602-9605.

Folstein, J. R., \& Van Petten, C. (2008). Influence of cognitive control and mismatch on the N2 component of the ERP: A review. Psychophysiology, 45(1), 152-170.

Fresco, D. M., Coles, M. E., Heimberg, R. G., Liebowitz, M. R., Hami, S., Stein, M. B., \& Goetz, D. (2001). The Liebowitz Social Anxiety Scale: A comparison of the psychometric properties of self-report and clinician-administered formats. Psychological Medicine, 31(6), $1025-1035$

Friesen, C. K., \& Kingstone, A. (1998). The eyes have it! Reflexive orienting is triggered by nonpredictive gaze. Psychonomic Bulletin \& Review, 5(3), 490-495.

Frith, C. D., \& Frith, U. (2006). The neural basis of mentalizing. Neuron, 50(4), 531-534.

Galfano, G., Sarlo, M., Sassi, F., Munafò, M., Fuentes, L. J., \& Umiltà, C. (2011). Reorienting of spatial attention in gaze cuing is reflected in N2pc. Social Neuroscience, 6(3), 257-269.

Goff, W. R., Allison, T., \& Vaughan, H. G. J. (1978). The functional neuroanatomy of event-related potentials. New York, NY: Academic Press.

Halladay, A. K., Bishop, S., Constantino, J. N., Daniels, A. M., Koenig, K., Palmer, K., ... Szatmari, P. (2015). Sex and gender differences in autism spectrum disorder: Summarizing evidence gaps and identifying emerging areas of priority. Molecular Autism, 6, 36.

Hayward, D. A., \& Ristic, J. (2017). Feature and motion-based gaze cuing is linked with reduced social competence. Scientific Reports, 7, 44221 .

Hietanen, J. K., Nummenmaa, L., Nyman, M. J., Parkkola, R., \& Hämäläinen, H. (2006). Automatic attention orienting by social and symbolic cues activates different neural networks: An fMRI study. NeuroImage, 33, 406-413.

Hillyard, S. A., \& Kutas, M. (1983). Electrophysiology of cognitive processing. Annual Review of Psychology, 34, 33-61.

Holmes, A., Bradley, B. P., Kragh Nielsen, M., \& Mogg, K. (2009). Attentional selectivity for emotional faces: Evidence from human electrophysiology. Psychophysiology, 46, 62-68.

Hommel, B., Pratt, J., Colzato, L., \& Godijn, R. (2001). Symbolic control of visual attention. Psychological Science, 12, 360-365.

Horley, K., Williams, L. M., Gonsalvez, C., \& Gordon, E. (2003). Social phobics do not see eye to eye: A visual scanpath study of emotional expression processing. Journal of Anxiety Disorders, 17(1), 33-44.

Horley, K., Williams, L. M., Gonsalvez, C., \& Gordon, E. (2004). Face to face: Visual scanpath evidence for abnormal processing of facial expressions in social phobia. Psychiatry Research, 127(1/2), 43-53.

Jarick, M., \& Kingstone, A. (2015). The duality of gaze: Eyes extract and signal social information during sustained cooperative and competitive dyadic gaze. Frontiers in Psychology, 6, 1423.
Johnson, M. H., Griffin, R., Csibra, G., Halit, H., Farroni, T., De Haan, M., . . . Richards, J. (2005). The emergence of the social brain network: Evidence from typical and atypical development. Development and Psychopathology, 17(3), 599-619.

Kayser, J., \& Tenke, C. E. (2003). Optimizing PCA methodology for ERP component identification and measurement: Theoretical rationale and empirical evaluation. Clinical Neurophysiology, 114(12), 2307-2325.

Klein, R. M. (2000). Inhibition of return. Trends in Cognitive Sciences, 4(4), 138-147.

Kobayashi, H., \& Kohshima, S. (1997). Unique morphology of the human eye. Nature, 387, 767-768.

Lasaponara, S., D’Onofrio, M., Pinto, M., Dragone, A., Menicagli, D., Bueti, D., . . . Doricchi, F. (2018). EEG correlates of preparatory orienting, contextual updating and inhibition of sensory processing in left spatial neglect. Journal of Neuroscience, 38(15), 3792-3808.

Leekam, S., Baron-Cohen, S., Perrett, D., Milders, M., \& Brown, S. (1997). Eye-direction detection: A dissociation between geometric and joint attention skills in autism. British Journal of Developmental Psychology, 15, 77-95.

Liebowitz, M. R. (1987). Social phobia. Modern Problems in Pharmacopsychiatry, 22, 141-173.

Lorenzo-Seva, U., \& ten Berge, J. M. F. (2006). Tucker's congruence coefficient as a meaningful index of factor similarity. Methodology: European Journal of Research Methods for the Behavioral and Social Sciences, 2, 57-64.

Luck, S. J., \& Hillyard, S. A. (1994). Electrophysiological correlates of feature analysis during visual search. Psychophysiology, 31(3), 291308.

Moukheiber, A., Rautureau, G., Perez-Diaz, F., Soussignan, R., Dubal, S., Jouvent, R., \& Pelissolo A. (2010). Gaze avoidance in social phobia: Objective measure and correlates. Behaviour Research and Therapy, 48(2), 147-151.

Mundy, P. (1995). Joint attention and social-emotional approach behavior in children with autism. Development and Psychopathology, 7(1), 63-82.

Murray, A. M., Nobre, A. C., \& Stokes, M. G. (2011). Markers of preparatory attention predict visual short-term memory performance. Neuropsychologia, 49(6), 1458-1465.

Näätänen, R., Gaillard, A. W., \& Mantysalo, S. (1978). Early selectiveattention effect on evoked potential reinterpreted. Acta Psychologica, 42(4), 313-329.

Näätänen, R., \& Michie, P. T. (1979). Early selective-attention effects on the evoked potential: A critical review and reinterpretation. Biological Psychology, 8(2), 81-136.

Nation, K., \& Penny, S. (2008). Sensitivity to eye gaze in autism: Is it normal? Is it automatic? Is it social? Development and Psychopathology, 20, 79-97.

Nixima, K., Fujimori, M., \& Okanoya, K. (2013). An ERP study of autistic traits and emotional recognition in non-clinical adolescence. Scientific Research Psychology, 4, 515-519.

Okada, T., Sato, W., Murai, T., Kubota, Y., \& Toichi, M. (2003). Eye gaze triggers visuospatial attentional shifts in individuals with autism. Psychologia, 46, 246-254.

Pfefferbaum, A., Ford, J. M., Weller, B. J., \& Kopell, B. S. (1985). ERPs to response production and inhibition. Electroencephalography and Clinical Neurophysiology, 60(5), 423-434.

Posner, M. I. (1980). Orienting of attention. The Quarterly Journal of Experimental Psychology, 32(1), 3-25.

Pritchard, W. S., Shappell, S. A., \& Brandt, M. E. (1991). Psychophysiology of N200/N400: A review and classification scheme. London, UK: Jessica Kingsley.

Ristic, J., \& Kingstone, A. (2012). A new form of human spatial attention: Automated symbolic orienting. Visual Cognition, 20(3), 244-264. 
Ristic, J., \& Landry, M. (2015). Combining attention: A novel way of conceptualizing the links between attention, sensory processing, and behavior. Attention, Perception, \& Psychophysics, 77(1), 36-49.

Ristic, J., Mottron, L., Friesen, C. K., Iarocci, G., Burack, J. A., \& Kingstone, A. (2005). Eyes are special but not for everyone: The case of autism. Cognitive Brain Research, 24(3), 715-718.

Seiss, E., Gherri, E., Eardley, A. F., \& Eimer, M. (2007). Do ERP components triggered during attentional orienting represent supramodal attentional control? Psychophysiology, 44(6), 987-990.

Semlitsch, H. V., Anderer, P., Schuster, P., \& Presslich, O. (1986). A solution for reliable and valid reduction of ocular artifacts, applied to the P300 ERP. Psychophysiology, 23(6), 695-703.

Senju, A., Tojo, Y., Dairoku, H., \& Hasegawa, T. (2004). Reflexive orienting in response to eye gaze and an arrow in children with and without autism. Journal of Child Psychology and Psychiatry, 45(3), 445-458.

Strom, J. C., \& Buck, R. W. (1979). Staring and participants' sex: Physiological and subjective reactions. Personality and Social Psychology Bulletin, 5(1), 114-117.

Suwazono, S., Machado, L., \& Knight, R. T. (2000). Predictive value of novel stimuli modifies visual event-related potentials and behavior. Clinical Neurophysiology, 111(1), 29-39.

Swettenham, J., Condie, S., Campbell, R., Milne, E., \& Coleman, M. (2003). Does the perception of moving eyes trigger reflexive visual orienting in autism? Autism: Mind and brain (pp. 89-107). New York, NY: Oxford University Press.
Tabachnik, B. G., \& Fidell, L. S. (2013). Using multivariate statistics (6th ed.). Boston, MA: Pearson.

Timoney, L. R., \& Holder, M. D. (2013). Emotional processing deficits and happiness. Dordrecht, Netherlands: Springer.

Tipples, J. (2002). Eye gaze is not unique: Automatic orienting in response to uninformative arrows. Psychonomic Bulletin \& Review, 9(2), 314-318.

Tucker, L. R. (1951). A method for synthesis of factor analysis studies (Personnel Research Section Report No. 984), Washington, DC: Department of the Army.

Vaughan, H. G. J., \& Arezzo, J. C. (1998). The neural basis of eventrelated potentials (Vol. 3, pp. 45-96). New York, NY: Elsevier.

Werling, D. M., \& Geschwind, D. H. (2013). Sex differences in autism spectrum disorders. Current Opinion in Neurology, 26(2), 146-153.

Woodman, G. F. (2010). A brief introduction to the use of event-related potentials in studies of perception and attention. Attention, Perception, \& Psychophysics, 72(8), 2031-2046.

Publisher's note Springer Nature remains neutral with regard to jurisdictional claims in published maps and institutional affiliations. 